\title{
Primary Intraventricular Brain Abscess Resulting in Isolated Dilation of the Inferior Horn and Unilateral Hydrocephalus
}

\author{
Joji Inamasu Shigeta Moriya Yushi Kawazoe Shinya Nagahisa \\ Mitsuhiro Hasegawa Yuichi Hirose \\ Department of Neurosurgery, Fujita Health University Hospital, Toyoake, Japan
}

\section{Key Words}

Brain abscess $\cdot$ Hydrocephalus $\cdot$ Inferior horn $\cdot$ Intraventricular

\begin{abstract}
Primary intraventricular brain abscesses are rare, and there are no established treatment guidelines for this condition. We report a case in which isolated ventricular dilatation and unilateral hydrocephalus developed after seemingly successful conservative management and which required surgical diversion of the cerebrospinal fluid. A 59-year-old woman presented to our emergency department with high-grade fever and headache. Brain magnetic resonance imaging (MRI) revealed abscesses in the bilateral posterior horn. Although surgical evacuation of the abscesses was considered, conservative management with antibiotics was selected because of the paucity of severe neurological deficits and the concern that an attempt to evacuate the intraventricular abscess might lead to inadvertent rupture of the abscess capsule and acute ventriculitis. Despite reduction in the abscess volume, the patient developed an altered mental status 4 weeks after admission. Follow-up MRI revealed isolated dilation of the left inferior horn, compressing the brainstem. Emergency fenestration of the dilated inferior horn was performed, and endoscopic observation revealed an encapsulated abscess with adhesion to the ventricular wall which was thought responsible for the ventricular dilation and unilateral hydrocephalus. Two weeks after the initial surgery, the unilateral hydrocephalus was treated by placement of a ventriculoperitoneal shunt. Eradication of the intraventricular brain abscesses without surgical evacuation may justify the conservative management of this patient. However, the possibility that earlier surgical evacuation might have prevented development of the isolated ventricular dilation cannot be denied. Additional
\end{abstract}

KARGER 125:s $\quad \begin{aligned} & \text { Joji Inamasu, MD, FACS } \\ & \text { Department of Neurosurgery, Fujita Health University Hospital } \\ & 1-98 \text { Kutsukake } \\ & \text { Toyoake } 470-1192 \text { (Japan) } \\ & \text { E-Mail inamasu@fujita-hu.ac.jp }\end{aligned}$


Inamasu et al.: Primary Intraventricular Brain Abscess Resulting in Isolated Dilation of the Inferior Horn and Unilateral Hydrocephalus

clinical experience is required to determine which treatment (surgical vs. conservative) is more appropriate in patients with primary intraventricular brain abscesses.

(C) 2015 S. Karger AG, Basel

\section{Introduction}

Rupture of a brain abscess into the cerebral ventricles is a devastating event, resulting in high mortality and morbidity. Patients with a ruptured abscess exhibit rapid neurological deterioration on account of acute ventriculitis [1]. Therefore, preemptive surgery to prevent rupture has been recommended for patients with an intraparenchymal brain abscess located near the ventricles [1]. In contrast, primary intraventricular brain abscesses have rarely been reported in the literature [2-4]. These lesions may exhibit clinical manifestations distinct from those of intraparenchymal brain abscesses. Because of their rarity, the treatment strategy for primary intraventricular brain abscesses has not been established. We report a case that developed isolated dilatation of the inferior horn and unilateral hydrocephalus, for which surgical diversion of the cerebrospinal fluid (CSF) was necessary.

\section{Case Report}

A 59-year-old woman with a history of thyroid cancer presented to our emergency department with a fever of $38.7^{\circ} \mathrm{C}$ and headache that had persisted for 2 days. She had received treatment for the thyroid cancer a year earlier, and there was no evidence of local or systemic metastasis. She was alert and oriented, and neurological examination revealed mild neck stiffness. Meningitis was suspected, and a CSF cell count obtained via a lumbar puncture showed 1,680 cells $/ \mathrm{mm}^{3}$ with predominantly polymorphonuclear cells. A complete blood count revealed a white blood cell count of 16,600 cells $/ \mathrm{mm}^{3}$. A brain magnetic resonance imaging (MRI) study revealed a slight dilation of the left inferior horn (fig. 1a) and low signal intensities in the bilateral posterior horn with uniform rim enhancement on T1-weighted images with gadolinium (fig. 1b). On diffusion-weighted images, the lesions were depicted as high signal intensities (fig. 1c). A diagnosis of intraventricular brain abscess was established, and the patient was admitted to our institution. Empirical antibiotics were initiated, comprising i.v. linezolid $600 \mathrm{mg}$ twice daily, i.v. meropenem $1 \mathrm{~g}$ three times daily, p.o. metronidazole $1,000 \mathrm{mg}$ four times daily, and p.o. sulfamethoxazole $1,600 \mathrm{mg} /$ trimethoprim $320 \mathrm{mg}$ twice daily. Although surgical evacuation of the abscesses by either craniotomy or endoscopy was considered, conservative management was selected because of the paucity of severe neurological deficits and the bilaterality of the lesions. The fever subsided within a week of antibiotic administration, and a follow-up MRI study at 2 weeks after admission showed mild abscess shrinkage (fig. 1d). No pathogens were isolated from the blood or CSF cultures. Approximately 4 weeks after admission, however, the patient became lethargic and aphasic. An MRI study performed after the deterioration revealed isolated dilation of the left inferior horn, compressing the brainstem (fig. 2a, b). Emergency transcortical fenestration of the dilated inferior horn was performed, and endoscopic observation of the lumen revealed an encapsulated abscess with adhesion to the ventricular wall (fig. 2c), which was thought to be responsible for the ventricular dilatation and unilateral hydrocephalus. Subsequently, an Ommaya reservoir was placed into the cavity (fig. $3 a, b)$. The patient became alert and oriented after surgery, and the dilated inferior horn showed gradual shrinkage (fig. 3c). Nevertheless, periodic CSF removal from the reservoir was necessary because of the unilateral 
Inamasu et al.: Primary Intraventricular Brain Abscess Resulting in Isolated Dilation of the Inferior Horn and Unilateral Hydrocephalus

hydrocephalus. After continuing antibiotics for another 2 weeks (6 weeks in total) and confirming the absence of bacteria in the CSF, the reservoir was replaced by a ventriculoperitoneal shunt (fig. 3d). The patient achieved full neurological recovery 6 months after admission, with disappearance of the abscesses on MRI. A written permission was obtained from the patient and her family for this report.

\section{Discussion}

Intraparenchymal brain abscesses located near the cerebral ventricles, for which urgent surgical intervention is required to prevent intraventricular rupture, are not infrequent in clinical practice [1]. In contrast, primary intraventricular brain abscess is rare, with less than 10 cases reported in the medical literature [2-4]. In the present case, the route of bacterial entry and propagation remains unclear, because there were no prodromal symptoms and the abscesses were already present when the patient presented to the emergency department. In most reported cases, intraventricular brain abscesses were located in the posterior horn of the lateral ventricle and were diagnosed after bacterial meningitis [2-4], suggesting that bacterial entry may be via the choroid plexus, which functions as the blood-CSF barrier [5]. Clinical symptoms in patients with primary intraventricular brain abscess may be more insidious and mild compared with the symptoms that occur after intraventricular rupture of an intraparenchymal brain abscess [2-4]. The lack of severity in the former condition may partly be due to abscess encapsulation, as seen in figure $2 \mathrm{~b}$. The pathogens causative of intraventricular brain abscess may be different from those responsible for intraparenchymal brain abscess: pathogens such as Nocardia and Toxoplasma, which cause opportunistic infection in immunocompromised patients, are often associated with the former condition [6-8]. In the present case, despite clinical response to sulfamethoxazole/trimethoprim, repeated cultures yielded negative results, and there was no evidence that these pathogens were causative.

There are no established guidelines or recommendations for the treatment of primary intraventricular brain abscess because of its rarity. In most previous reports, surgical evacuation of the abscess was performed via craniotomy [2-4]. Unlike previously reported cases in which an abscess was located unilaterally, the present case harbored bilateral lesions, which may have necessitated bilateral craniotomy. Good response to antibiotics with chronological abscess shrinkage was an additional reason for continuation of conservative management. In addition to craniotomy, endoscopic abscess evacuation may have been another option; the efficacy of the neuroendoscope in the evacuation of a brain abscess that has already ruptured into the lateral ventricles has been reported [9]. However, we selected conservative management mostly because of the concern that an attempt to evacuate the intraventricular abscess endoscopically may lead to inadvertent rupture of the abscess capsule and dispersion of purulent material into the entire ventricles.

There is little information on the mechanism by which antibiotics reach an intraventricular brain abscess. I.v. antibiotics may be delivered less efficiently in intraventricular brain abscesses compared with their intraparenchymal counterparts considering the scarcer blood supply in the former. Therefore, we included linezolid, which has a good CSF penetration profile, in the antibiotic regimen [10]. The finding that the intraventricular brain abscess was eradicated without surgical evacuation may justify conservative management. However, our patient developed isolated dilatation of the inferior horn, which may have been caused by adhesion and obstruction of the CSF pathway subsequent to prolonged infection [11]. Moreover, choroid plexitis and resultant CSF overproduction may also have contributed to 
Inamasu et al.: Primary Intraventricular Brain Abscess Resulting in Isolated Dilation of the Inferior Horn and Unilateral Hydrocephalus

the development of ventricular dilatation and unilateral hydrocephalus [12]. Therefore, the possibility that earlier surgical evacuation of the intraventricular brain abscesses might have prevented the development of isolated ventricular dilation cannot be denied. Further clinical experience is necessary to determine which treatment (surgical vs. conservative) is more appropriate in such cases.

\section{References}

1 Inamasu J, Kuramae T, Tomiyasu K, Nakatsukasa M: Fulminant ependymitis following intraventricular rupture of brain abscess. J Infect Chemother 2011;17:534-537.

-2 Lambo A, Nchimi A, Khamis J, Lenelle J, Francotte N: Primary intraventricular brain abscess. Neuroradiology 2003;45:908-910.

- Gadgil N, Chamoun RB, Gopinath SP: Intraventricular brain abscess. J Clin Neurosci 2012;19:1314-1316.

4 Han KT, Choi DS, Ryoo JW, Cho JM, Jeon KN, Bae KS, You JJ, Chung SH, Koh EH, Park KJ: Diffusion-weighted MR imaging of pyogenic intraventricular empyema. Neuroradiology 2007;49:813-818.

5 Engelhardt B, Wolburg-Buchholz K, Wolburg H: Involvement of the choroid plexus in central nervous system inflammation. Microsc Res Tech 2001;52:112-129.

-6 Terrier A, Jourdan C, Remond J, Vighettho A, Peloux A, Naous H, Artru F: Unilateral hydrocephalus caused by abscess of the choroid plexus. Rev Neurol (Paris) 1992;148:234-236.

7 Mogilner A, Jallo GI, Zagzag D, Kelly PJ: Nocardia abscess of the choroid plexus: clinical and pathological case report. Neurosurgery 1998;43:949-952.

8 Hagiwara E, Nath J: Choroid plexitis in a case of systemic nocardiosis. Emerg Radiol 2007;14:337-343.

-9 Nishizaki T, Ikeda N, Nakano S, Sakakura T, Abiko M, Okamura T: Successful neuroendoscopic treatment of intraventricular brain abscess rupture. Clin Pract 2011;1:e52.

10 Boak LM, Li J, Spelman D, du Cros P, Nation RL, Rayner CR: Successful treatment and cerebrospinal fluid penetration of oral linezolid in a patient with coagulase-negative Staphylococcus ventriculitis. Ann Pharmacother 2006;40:1451-1455.

11 Miyake H, Ohta T, Oi S, Kajimoto Y, Ogawa D: Isolated dilation of the trigono-inferior horn: four case reports. Neurol Med Chir (Tokyo) 2000;40:179-185.

$\checkmark 12$ Bourgouin PM, Melançon D, Carpenter S, Tampieri D, Ethier R: Hydrocephalus and prominence of the choroid plexus: an unusual computed tomographic presentation of cerebral toxoplasmosis in AIDS. Can Assoc Radiol J 1992;43:55-59. 
Inamasu et al.: Primary Intraventricular Brain Abscess Resulting in Isolated Dilation of the Inferior Horn and Unilateral Hydrocephalus

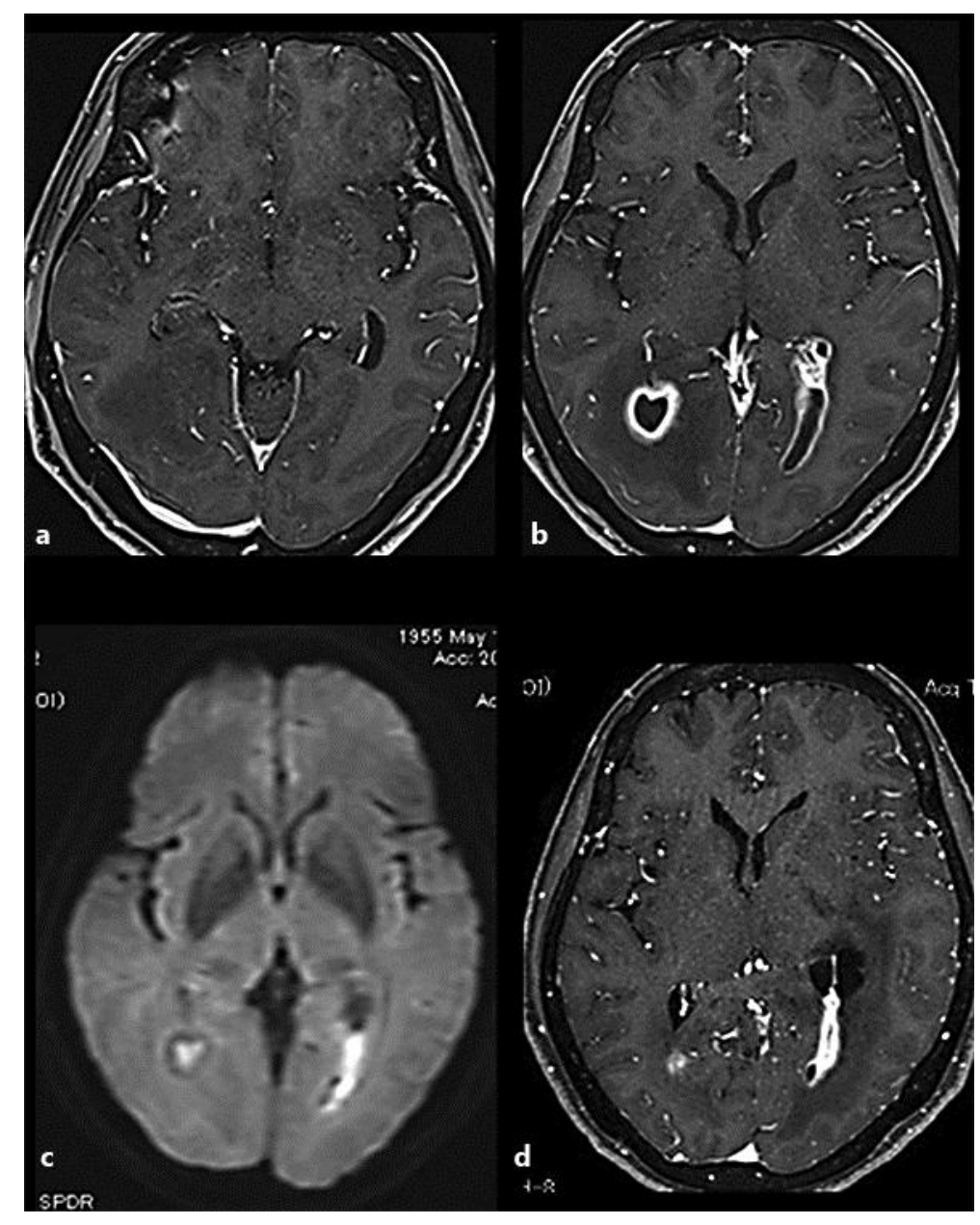

Fig. 1. MRI on admission showing a slight dilation of the left inferior horn (a) and low signal intensities in the bilateral posterior horn with uniform rim enhancement by gadolinium (b). On diffusion-weighted imaging, the lesions were depicted as high signal intensities (c). Contrast-enhanced T1-weighted imaging performed 2 weeks after admission showing mild shrinkage of the abscesses (d). 


\section{Case Reports in Neurology}
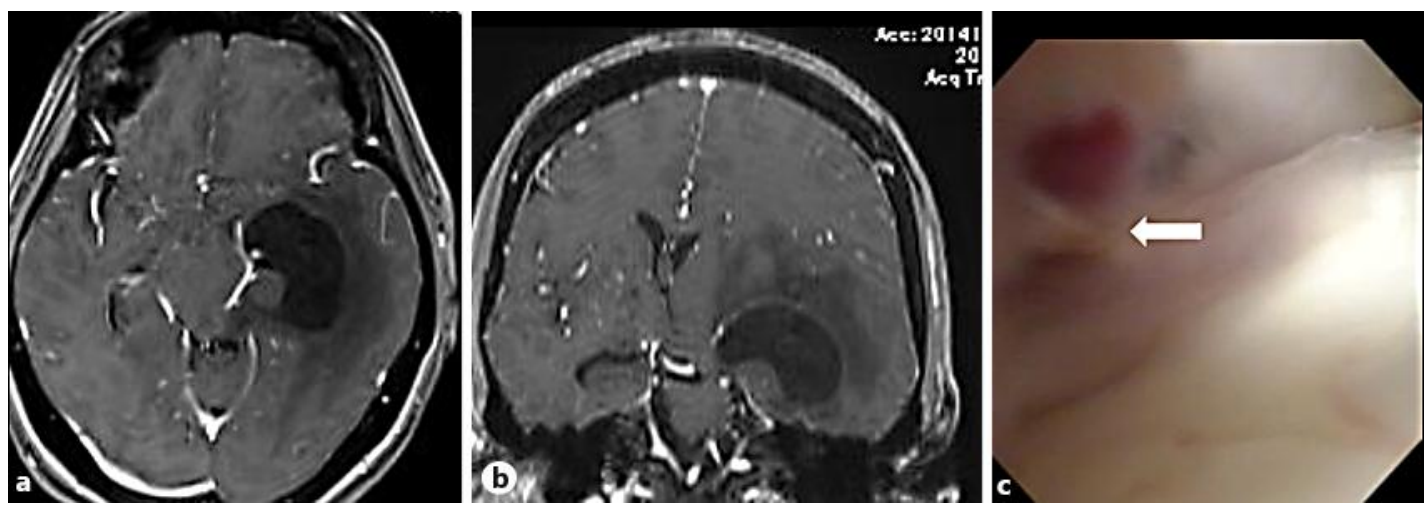

Fig. 2. MRI performed 4 weeks after admission showing marked dilatation of the left inferior horn (a axial view; b coronal view). Endoscopic fenestration was performed, showing encapsulated abscesses (c arrow) and adhesion to the ventricular wall.

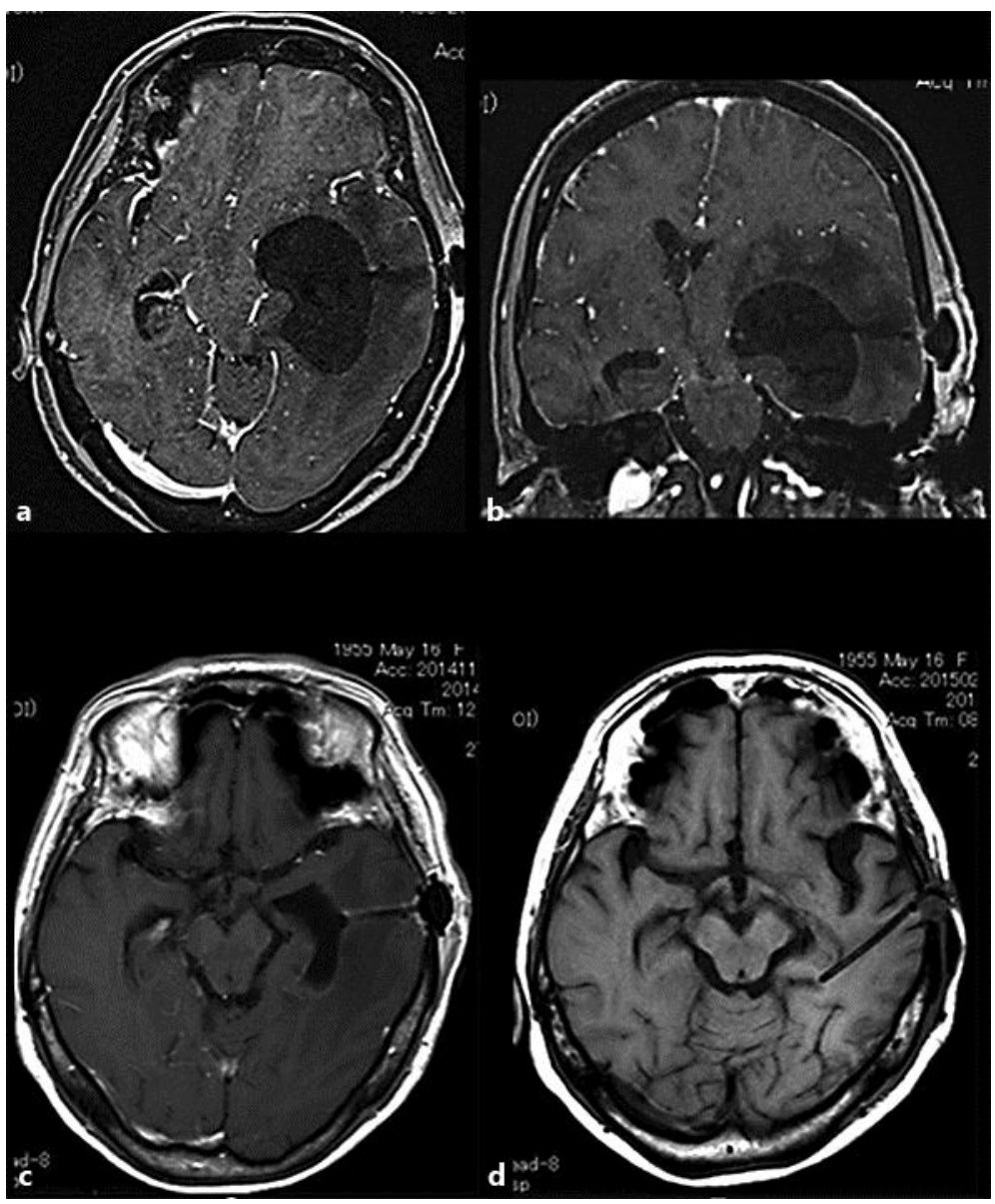

Fig. 3. MRI performed after endoscopic fenestration and placement of the Ommaya reservoir showing the trajectory from the brain surface to the dilated inferior horn (a axial view; b coronal view). MRI performed after removal of the CSF via the reservoir showing shrinkage of the dilated inferior horn (c). MRI performed after a ventriculoperitoneal shunt surgery showing the trajectory of a ventricular catheter and the inferior horn which returned to a normal configuration (d). 